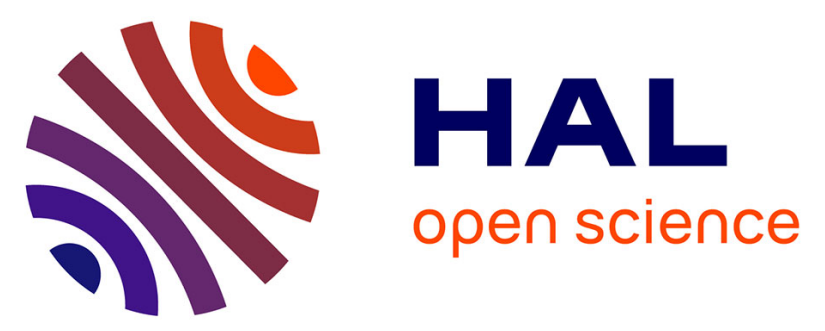

\title{
HIFUNet: Multi-class Segmentation of Uterine Regions from MR Images Using Global Convolutional Networks for HIFU Surgery Planning
}

Chen Zhang, Huazhong Shu, Guanyu Yang, Faqi Li, Yingang Wen, Qin Zhang, Jean-Louis Dillenseger, Jean-Louis Coatrieux

\section{To cite this version:}

Chen Zhang, Huazhong Shu, Guanyu Yang, Faqi Li, Yingang Wen, et al.. HIFUNet: Multiclass Segmentation of Uterine Regions from MR Images Using Global Convolutional Networks for HIFU Surgery Planning. IEEE Transactions on Medical Imaging, 2020, 39 (11), pp.3309-3320. 10.1109/tmi.2020.2991266 . hal-02569014

\section{HAL Id: hal-02569014 \\ https://hal.science/hal-02569014}

Submitted on 10 May 2020

HAL is a multi-disciplinary open access archive for the deposit and dissemination of scientific research documents, whether they are published or not. The documents may come from teaching and research institutions in France or abroad, or from public or private research centers.
L'archive ouverte pluridisciplinaire HAL, est destinée au dépôt et à la diffusion de documents scientifiques de niveau recherche, publiés ou non, émanant des établissements d'enseignement et de recherche français ou étrangers, des laboratoires publics ou privés. 


\title{
HIFUNet: Multi-class Segmentation of Uterine Regions from MR Images Using Global Convolutional Networks for HIFU Surgery Planning
}

\author{
Chen Zhang, Huazhong Shu, Senior Member, IEEE, Guanyu Yang, Faqi Li, Yingang Wen, Qin Zhang, \\ Jean-Louis Dillenseger, Senior Member, IEEE, and Jean-Louis Coatrieux Fellow, IEEE
}

\begin{abstract}
Accurate segmentation of uterus, uterine fibroids, and spine from MR images is crucial for high intensity focused ultrasound (HIFU) therapy but remains still difficult to achieve because of 1) the large shape and size variations among individuals, 2) the low contrast between adjacent organs and tissues, and 3) the unknown number of uterine fibroids. To tackle this problem, in this paper, we propose a large kernel Encoder-Decoder Network based on a 2D segmentation model. The use of this large kernel can capture multi-scale contexts by enlarging the valid receptive field. In addition, a deep multiple atrous convolution block is also employed to enlarge the receptive field and extract denser feature maps. Our approach is compared to both conventional and other deep learning methods and the experimental results conducted on a large dataset show its effectiveness.
\end{abstract}

Index Terms-Encoder-Decoder, Global convolutional networks, HIFU, MR images, Segmentation, Uterine fibroids

\section{INTRODUCTION}

$\mathbf{U}$ TERINE fibroids are benign tumors, common and present in up to $25 \%$ of women [1]. High intensity focused ultrasound (HIFU) is a new noninvasive surgery method for treating uterine fibroids. Magnetic Resonance (MR) image is clinically used for their diagnosis and the guidance of the HIFU procedure. The segmentation of uterus and uterine fibroids is a prerequisite step for the planning of HIFU treatment. However, the segmentation of the spine is also important in order to avoid any injury to the spinal cord. Manual delineation of the uterus, fibroids, and spine is a timeconsuming, tedious task and subject to intra-expert and interexpert variability during both pre- and post-treatment. Thus,

C. Zhang, H. Shu and G. Yang are with the Laboratory of Image Science and Technology, Southeast University, Nanjing 210096, China, also with the Centre de Recherche en Information Biomedicale Sino-Français, F-35000 Rennes, France, and also with the Key Laboratory of Computer Network and Information Integration, Ministry of Education, Southeast University, Nanjing 210096, China (e-mail: chenzhang@seu.edu.cn; shu.list@seu.edu.cn; yang.list@seu.edu.cn).

F. Li is with the State Key Laboratory of Ultrasound Engineering in Medicine, College of Biomedical Engineering, Chongqing Medical University, Chongqing 400016, China (e-mail: lifq@cqmu.edu.cn).

Y. Wen is with National Engineering Research Center of Ultrasound Medicine, Chongqing 401121, China (e-mail: wenyg@haifu.com.cn).

Q. Zhang is with Chongqing Haifu Medical Technology Co. Ltd. Chongqing 401121, China (e-mail: zhangq@haifu.com.cn).

J.-L. Dillenseger and J.-L. Coatrieux are with the Centre de Recherche en Information Biomedicalee Sino-Français, 35042 Rennes, France, also with Univ Rennes, Inserm, LTSI - UMR 1099, F-35000 Rennes, France (e-mail: jean-louis.dillenseger@univ-rennes1.fr; jean-louis.coatrieux@univrennes1.fr).

(Corresponding author: Huazhong Shu.).
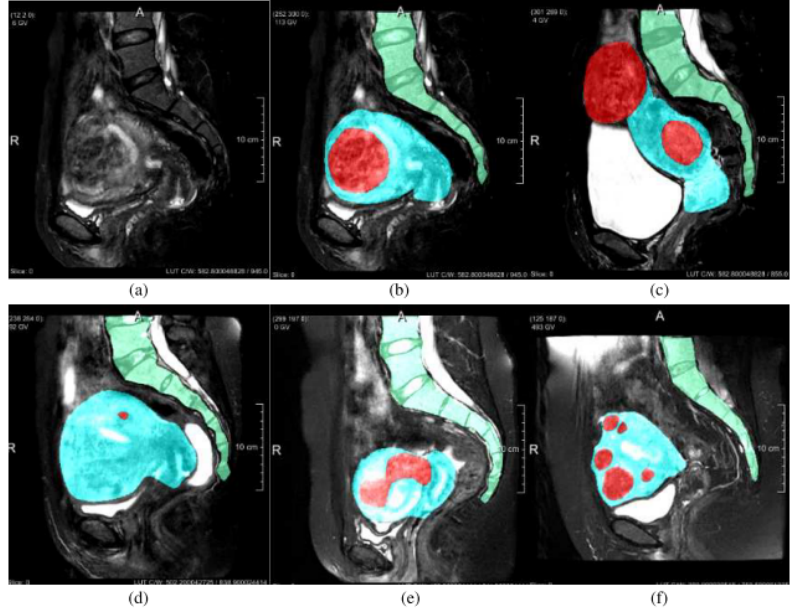

Fig. 1. MR images of uterus regions in different patients. Red denotes the fibroids, blue the uterus, and green the spine. (a) Patient 71 slice 14 of raw MR image. (b-f) The labeled images of Patient 71 slice14, Patient 84 slice14, Patient 93 slice12, Patient 26 slice12, Patient 8 slice13. We can observe 1) large shape and size variations among individuals; 2) a low contrast between adjacent organs and tissues; 3) highly variable uterine fibroids numbers and shapes.

an automatic and accurate segmentation method capable to extract all these structures is of great importance.

Such an objective is challenging because of 1) large shape and size variations among individuals. As it is shown in Fig. 1, uterine and fibroids are highly variable in different patients; 2) a low contrast between adjacent organs and tissues. The contrast among uterus and uterine fibroids is quite low, so the boundaries between organs are difficult to distinguish; 3) the number of uterine fibroids and their shapes are unknown. These issues are illustrated in Fig. 1. Due to the above reasons, the existing methods dealing with uterine fibroid segmentation are often applied after treatment, while the pre-treatment is still performed manually by an operator to mark uterus, fibroids and surrounding organs. Therefore, in order to facilitate the development of a treatment plan, a preoperative segmentation is required.

In recent years, deep learning (DL) methods have been widely used in medical image segmentation [2], [3], [4]. However, they have to face the overall complexity of the scenes under study. We propose here to derive comprehensive anatomical information through a global convolutional network (GCN) module based on a large valid receptive field and 
deep multiple atrous convolutions (DMAC) for hierarchically structuring the information. By doing so, the performance in locating and classifying the structures of interest can be improved. Such semantic segmentation can be built upon the Encoder-Decoder architecture already widely utilized. Inspired by Fully Convolutional Network (FCN) [5] which was initially designed for image classification, U-Net was proposed for medical image segmentation by Ronneberger et al. [6] where the pooling operators in FCN are replaced by upsampling operators so that the output resolution can be retained at the same size as the input. The state-of-the-art results of U-Net in segmenting medical images, especially with small training dataset, show a promising ability of this Encoder-Decoder architecture. Basically, the Encoder aims to capture features and reduce the spatial dimensions while the Decoder aims to recover the object details and spatial dimension. Therefore, in order to improve the performance of image segmentation, more high-level features need to be automatically captured in the encoder and more spatial information can be saved in the decoder.

The U-Net was later extended in order to tackle different problems. Cicek et al. [7] modified the initial U-Net architecture by replacing all 2D operations with their 3D counterparts. Milletari et al. [8] presented a novel 3D segmentation approach (called V-Net) that leverages the power of a fully convolutional neural network based on the Dice coefficient for processing volumetric medical images such as MR images. In addition, in contrast with 3D U-Net, the V-net formulates each stage by using a residual function which can accelerate the convergence rate. Many other U-Net based segmentation schemes have been further reported for retinal vessels, liver and tumors in CT scans, ischemic stroke lesion, intervertebral disc and pancreas [9], [10], [11], [12], [13], [14], [15], [16], [17], [18], [19], [20].

The U-Net shows a good segmentation performance with the usage of skip connections which can concatenate two feature maps of the same size in the corresponding parts of the encoder and decoder. The concatenated feature maps contain the information from both high and low levels, thus achieving feature fusion under different scales to improve the accuracy of model results. Even so, the complex anatomical scene involved in our HIFU therapy application remains a challenge. Large valid receptive fields play an important role in global scene observation. Global convolutional network [21] enables dense connections within a large region by using spatial decomposed convolution with a large kernel. It can capture multi-scale context cues with less computational cost than a general convolution with a large kernel. Therefore, we introduce layer-by-layer the GCN which has an efficient kernel parameter number to enlarge the receptive field in our Encoder-Decoder architecture.

In addition, getting the hierarchical structural information can help to provide more contextual information at various levels by using atrous convolutions. The key element of this method is to insert holes into the convolution kernels, which allows preserving the resolution and enlarging the receptive field. Recently, atrous convolution has been widely used in many deep learning architectures. DeepLab [22], based on
FCN and atrous convolutions, maintains the receptive field unchanged. Besides, in order to get a better object segmentation at multiple scales, in DeepLabV2 [23], Chen et al. Proposed a module called atrous spatial pyramid pooling (ASPP) which uses multiple parallel atrous convolutional layers with different sampling rates. The use of atrous convolutions preserves the spatial resolution of the final map and thus leads to higher performance when compared to most methods in EncoderDecoder schemes. DeepLabV3+ [24] combines the advantages of Xception [25] and Encoder-Decoder, which employs DeepLabV3 [26] as the encoder.

However, the uncertainty regarding the location, the numbers and the sizes of uterine fibroids leads to an increase of complexity for segmentation and many existing deep learning segmentation models lack using features from different levels efficiently. Subsequently, in some cases, the targets can be segmented incorrectly. More effective feature extraction approaches are required for uterine fibroid segmentation.

Motivated by the above discussions and ResNet [27] structures, we propose a novel network named HIFUNet to segment uterus, uterine fibroids and spine automatically. The main contributions of the paper can be summarized as follows:

1) To address the segmentation errors (i.e., classifying uterine neck as uterine fibroid because of insufficient receptive field), we introduce a GCN module able to enlarge the receptive field effectively.

2) We integrate the GCN and DMAC to further extract context-based semantic information and generate more abstract features for large scaled uterine fibroid.

3) The proposed HIFUNet behaves similarly to clinical experts and, as it will be shown through a large number of experiments, performs better than many existing semantic segmentation networks.

4) The segmentation of the uterus and uterine fibroids is, to the best of our knowledge, the first methodological attempt using convolutional neural networks in HIFU therapy. The inclusion of the spine segmentation, a critical organ in HIFU therapy, is another major feature of our approach.

The structure of this paper is as follows: In Section II, we describe up-to-date related work. Our solution is then introduced in section III. Our experiments are reported in Section IV, including performance comparisons with conventional and other deep learning methods. In Section V, we draw some conclusions and perspectives.

\section{RELATED WORK}

We sketch here the conventional methods proposed so far for segmenting the uterus and uterine fibroids and we review the state-of-the-art MR image segmentation methods based on $\mathrm{CNN}$ architectures.

\section{A. Conventional methods of uterus and uterine fibroid seg- mentation}

Very few contributions have been reported for segmenting uterus and uterine fibroids from MR images. The main methods are summarized below: 
Approaches based on level-set: Ben-Zadok et al. [28] presented an interactive level set segmentation framework that allows user feedback. It is a semi-automatic method where the users have to select seed-points. Khotanlou et al. [29] proposed a two-stage method combining the region-based level set [30] and the hybrid Bresson methods [31]. Yao et al. [32] employed a method based on a combination of fast marching level-set and Laplacian level set.

Approaches based on Fuzzy C-Means (FCM): Fallahi et al. [33] segmented the uterine fibroids by combining a fuzzy C-Means method with some morphological operations. Later, on the basis of [33], a two-step method [34] was proposed by employing a Modified Possibilistic Fuzzy C-Means (MPFCM) [35] in a second step.

Approaches based on region-growing: Militello et al. [36] used a semi-automatic approach based on region-growing and reported a quantitative and qualitative evaluation of the HIFU treatment by providing the 3D model of the fibroid area. Rundo et al. [37] presented a two-phase method where the first phase is an automatic seed-region selection and region detection while the second one is aimed at uterine fibroid segmentation.

Other mixed methods: Antila et al. [38] designed an automatic segmentation pipeline without user input. They applied the active shape model (ASM) to get the deformed surface, and classified PV (perfused volume: the untreated tissue) and NPV (non-perfused volume: the treated tissue) by an expectation maximization (EM) algorithm. Militello et al. [39] proposed a novel fully automatic method based on the unsupervised Fuzzy C-Means clustering and iterative optimal threshold selection algorithms for uterus and fibroid segmentation.

Recently, Rundo et al. [40] evaluated the above mentioned two computer-assisted segmentation methods [37], [39] and provided a quantitative comparison on segmentation accuracy in terms of area-based and distance-based metrics. Their results show that both methods remarkably outperform the other ones.

However, there are still some limitations and drawbacks in the conventional methods and a fully-automatic and accurate method, able to reduce or even to remove pre-processing/postprocessing procedures as well as the interventions of the medical physicists, is still expected. For this purpose, a detailed comparison between the methods reported in [37] and [39] and our method will be shown in Section IV.

\section{B. Deep Learning Methods of MR Image Segmentation}

Only a few attempts have been reported for the uterus segmentation using CNN-based methods. Kurata et al. [41], [42] evaluated the clinical feasibility of fully automatic uterine segmentation on T2-weighted MR images based on an optimized U-Net. The segmentation of uterus in this research was focused on the staging of uterine endometrial cancer and on estimating the extent of tumor invasion to the uterine myometrium. To the best of our knowledge, there is no literature published on the uterine fibroid segmentation using CNN-based methods. Even so, it is important to highlight that many innovative deep learning methods have been proposed for MR image processing [43], [44]. The most common applications concern segmentation of organs, substructures, or lesions, often as a preprocessing step for feature extraction and classification. Deep learning methods for MR image segmentation can be divided into two different categories.

DL based on image patches: Features are extracted from a local patch for every voxel using convolutional layers. These features are then classified with a fully connected neural network to obtain a label for every voxel. This method is for instance widely used in brain tumor [45], white matter segmentation in multiple sclerosis patients [46], normal components of brain anatomy [47] and rectal cancer segmentation [48]. However, such methods have some disadvantages. The main problem is that their computational efficiency is very low because they have to process overlapping parts of the image. Another disadvantage is that each voxel is segmented based on a finite size context window, ignoring the broader context. In some cases, more global information may be needed to properly assign these labels to pixels or voxels.

Fully convolutional neural network (FCNN): In this case, the entire image or a large portion is processed, the output being a segmentation result instead of a label of a single pixel or voxel. Such an approach solves the shortcomings of the former method and improves the efficiency of the algorithm. Many architectures can be considered for segmentation among which, as mentioned in Section I, encoder-decoder ones such as U-Net and its modified versions [9]-[20]. For MR images, we refer to [43] for a full survey. Zhang et al. [49] used CNN for segmenting the infant brain tissues by combining $\mathrm{T} 1, \mathrm{~T} 2$, and FA images into white matter (WM), gray matter (GM), and cerebrospinal fluid (CSF). Brain tumor segmentation was addressed in [50]. Avendi et al. [51] associated DL algorithms with deformable models for the left ventricle segmentation of the heart. Milletari et al. [8] proposed a 3D image segmentation based on a volumetric, fully convolutional, neural network. Their CNN was trained end-to-end on MR image volumes depicting the prostate and learned to predict segmentation for the whole volume at once. Some universal architectures were also proposed (for instance CE-Net by $\mathrm{Gu}$ et al. [52]) to address different clinical applications.

However, our target presents significant differences with these examples (i.e. brain, prostate, and heart). The deformation of the uterus shape is very large among the patients. The uterus position is also varying a lot. The high number of surrounding organs together with their similarity in tissue features makes more challenging the segmentation. In addition, different kinds of uterine fibroids (such as subseries fibroids, submucosal fibroids, intramural uterine fibroid tumors, pedunculated leiomyomas, and parasitic uterine fibroids) may be located in different regions of the uterus, and the gray level of these fibroids are affected by the signal intensity and other experimental factors. All these considerations have guided the design of our approach.

\section{METHOD}

To accurately segment the uterus, uterine fibroids and spine from the raw MR images, we propose an Encoder-Decoder 


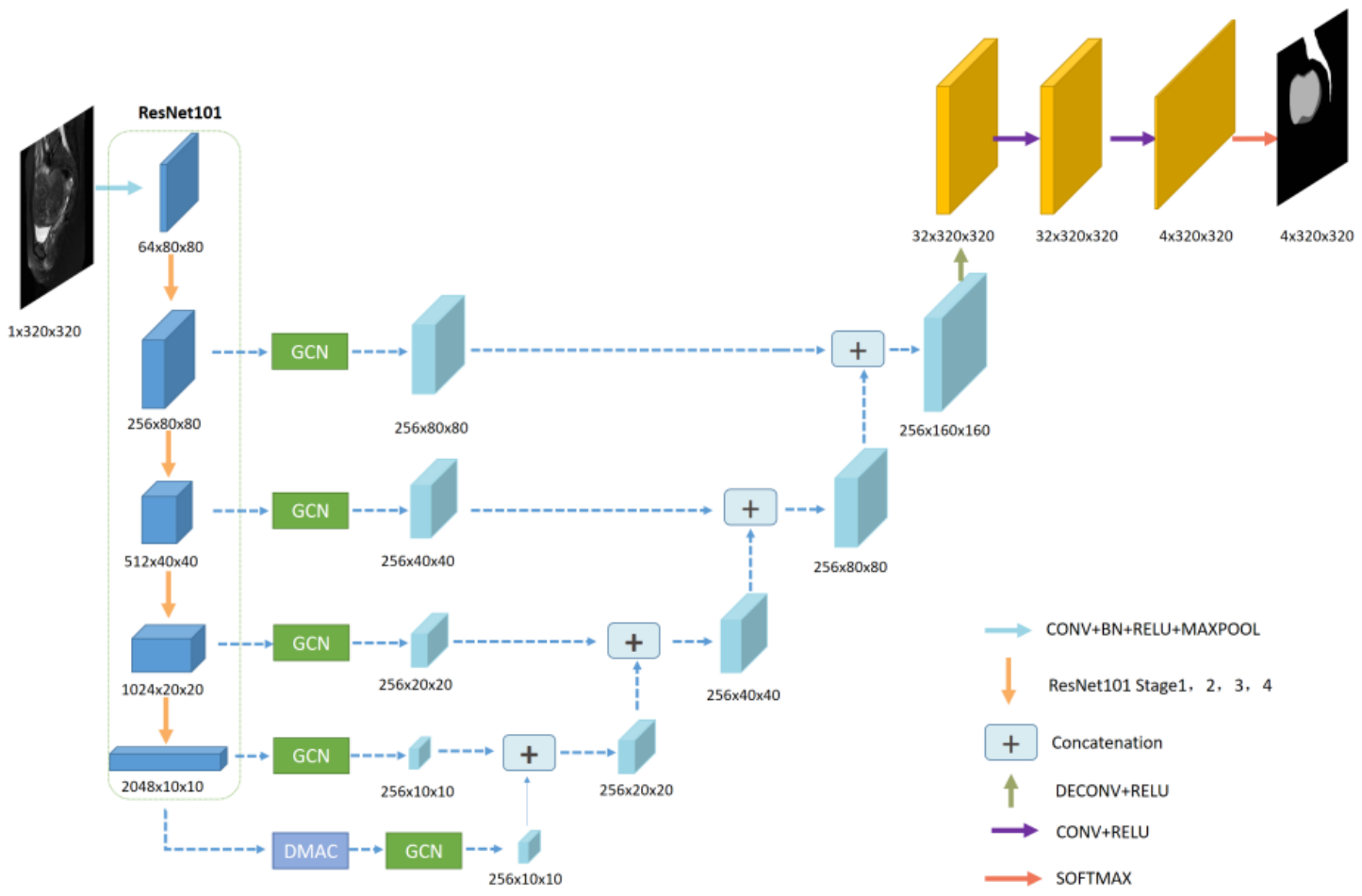

Fig. 2. The architecture of our proposal network (HIFUNet). The network consists of Resnet101 backbone, GCN module, DMAC module, upsampling layers, concatenation layers, and an output layer. The parameters and sizes of output features in different layers are presented in different colors

global convolutional network. The whole pipeline is illustrated in Fig. 2. This network (called HIFUNet) consists of three major parts: the feature encoder module, the feature extractor part (with the global convolution network and deep multiple atrous convolutions) and the feature decoder module.

\section{A. Encoder Module}

The encoder part uses pre-trained ResNet101 [27]. In [53], the authors demonstrated that the use of residual connections promotes information propagation both forward and backward, so it helps to improve significantly both the training speed and the performance. Because we have only one channel in our raw 2D input image, we change the original first portion which forms three input channels to one channel and we obtain 64 channels after the first Conv1. Then, four feature extracting blocks are employed. The first, second, third, and fourth stages contain 3, 4, 23, and 3 bottlenecks respectively and each block has no average pooling layer or fully connected layers.

\section{B. Global Convolution Network}

The current trend in architecture design goes toward stacking small convolution kernels because this option is more efficient than using a large convolution kernel with the same amount of computation. However, considering that semantic segmentation tasks require pixel-by-pixel segmentation prediction, Peng et al. [21] proposed a global convolutional network to improve the accuracy of classification and localization simultaneously. In GCN, a fully-convolutional layer is adopted to replace the global pooling layer in order to keep the localization information. Besides, large kernels are introduced

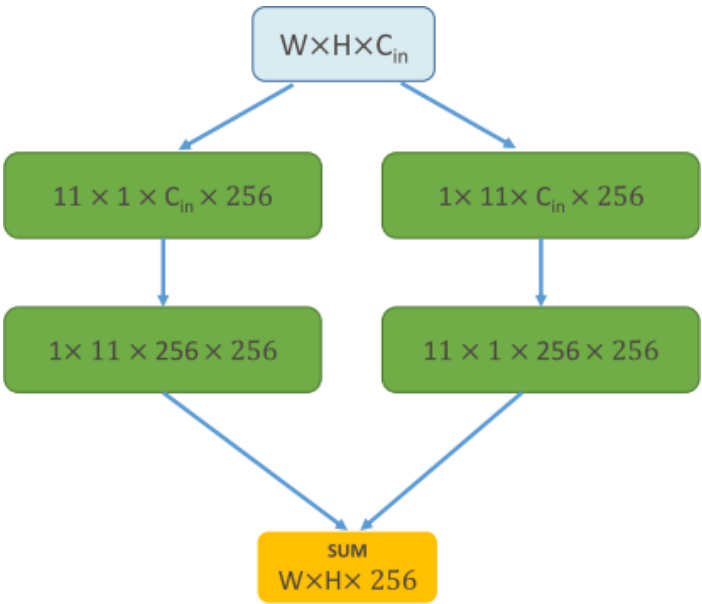

Fig. 3. Global Convolutional Network

to increase the valid receptive field (VRF). However, using a large kernel or a global convolution directly is inefficient. To further improve the computational efficiency, GCN uses a combination of two large 1D convolutional kernels to replace a single 2D kernel for the skip-connector layer. The architecture of GCN is shown in Fig. 3. The kernel size we use in our segmentation approach is $11 \times 11$.

\section{Deep Multiple Atrous Convolutions}

Atrous convolutions solve the problem of reduced resolution caused by the Deep Convolutional Neural Networks (DCNNs) while adjusting the receptive field of the filter. Fig. 4 illustrates the atrous convolution. The main idea of atrous dilation 


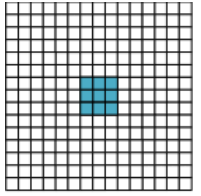

Dilation rate $=$

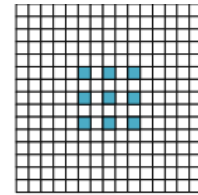

Dilation rate $=2$

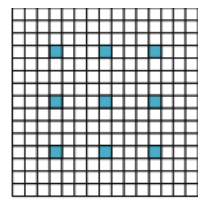

Dilation rate $=4$
Fig. 4. Atrous convolutions with $3 \times 3$ kernel (blue blocks) and rates 1,2 or

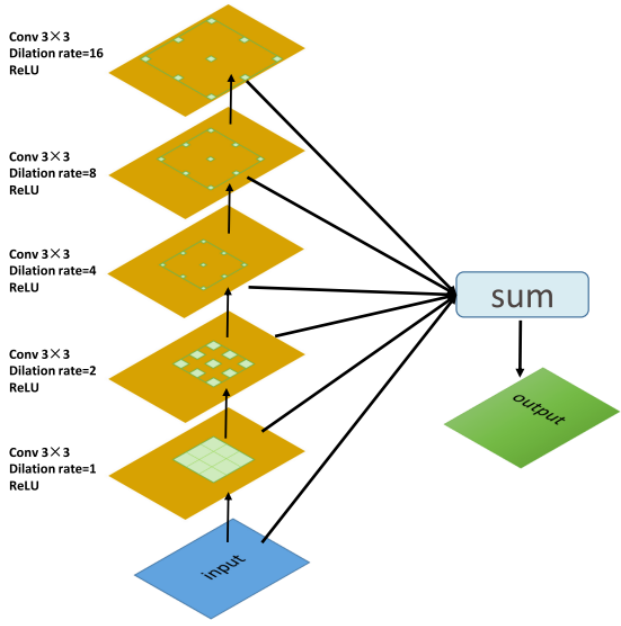

Fig. 5. Deep multiple atrous convolutions (DMAC) consist of five atrous convolutional layers

rate convolution is to insert "holes" (zeros) between pixels in convolutional kernels to increase the image resolution, enabling thus dense feature extraction in DCNNs. The atrous convolution was initially proposed to efficiently compute the undecimated wavelet transform [54] and the wavelet decomposition [55] in the atrous scheme. In recent years, atrous convolution has been widely used in tasks such as semantic segmentation and object detection. The Deeplab series [22]-[24], [26] and dense upsampling convolution (DUC) [56] made thorough studies of atrous convolution. Fig. 5 shows our proposed deep multiple atrous convolution scheme to achieve multi-scale representations. We implement five convolutional layers with $3 \times 3$ kernels with different sampling rates to extract the different features. Finally, we fuse all features with the input image to generate the final result.

When compared to the conventional network structure, our deep multiple atrous convolutions can extract multiple features and provide receptive fields of multiple sizes. It can be noticed that the architecture of our atrous convolution scheme adopts a serial frame instead of a parallel structure such as Inception and Atrous Spatial Pyramid Pooling (ASPP). We employ the DMAC block in the final layer of the encoder and this way more abstract information can be exploited. Within the DMAC block, as the layer is deeper, the dilation rate is getting larger. Because of the kernel discontinuity, not all pixels are used for calculation, so more atrous rate convolutions can compensate for the uncalculated information in the serial structure, which can increase the receptive field effectively. Besides, different sizes of atrous rates can help to extract different sized targets (from small fibroids to large organs like uterus or spine). The serial structure can get global distribution information from various scales of atrous convolution. The final step sums up as the output the abstract information extracted from the multiple layers. This output is then sent to the decoder phase in order to recover the object details and spatial dimensions. Therefore, in order to improve the performance of image segmentation, more low and high-level features are automatically captured in the encoder.

\section{Decoder Module}

The decoder module mainly uses the concatenation operation to fuse the multi-scale features. U-Net concatenates the downsampling feature maps with the corresponding upsampling feature maps. Here, this concatenation is performed between two neighboring feature maps after the GCN modules and this from the bottom to the top. After four concatenation operations, the image scale increases from $1 / 32$ to $1 / 2$ of the input image size. Then, we use a deconvolution operation to enlarge the image scale to the initial size and to restore features with more detailed information. Finally, the output mask is obtained after applying two convolution operations and softmax. As illustrated in Fig. 2, the decoder module mainly includes four concatenation operations $(\mathrm{a} 1 \times 1$ convolution, a $4 \times 4$ transposed convolution, and two $3 \times 3$ convolutions consecutively). Then, the feature decoder module outputs a mask with the same size as the original input.

\section{E. Loss Function}

The HIFUNet can be trained by minimizing the crossentropy error between its prediction result and the groundtruth. The loss function is defined as

$$
L=\sum_{i \in \Omega} y_{c_{i}} \log \left(p_{c_{i}}\right)+\left(1-y_{c_{i}}\right) \log \left(1-p_{c_{i}}\right)
$$

where $p_{c_{i}}$ denotes the predicted probability of $c$-th class for pixel $i$ in the predicted result $p, y_{c_{i}} \in\{0,1\}$ is the corresponding ground-truth value. If $y_{c_{i}}=1$, it means that Pixel $i$ belongs to the $c$-th class. If $y_{c_{i}}=0$, it means that pixel $i$ does not belong to the $c$-th class. $c=0$ denotes the background, $c=1$ denotes the uterus, $c=2$ denotes the uterine fibroids while $c=3$ denotes the spine. $\Omega$ denotes the space of the predicted result of $p$ and the ground-truth $y$. By minimizing the loss function on a training database, the parameters of HIFUNet can be optimized. Then the trained HIFUNet can be applied for automated uterus, uterine fibroids and spine segmentation on different datasets

\section{F. Further Discussion}

The main difference between our HIFUNet and other stateof-the-art deep learning networks including GCN, HRNet, UNet, CE-Net, AttentionUNet, and LEDNet is summarized as follows:

- GCN uses large kernels to enlarge the effective receptive field which can help classify different objects. Different from $\mathrm{GCN}$, in order to exploit more abstract information, HIFUNet adds an original DMAC block which improves 
the accuracy of segmentation of key parts such as the cervix and minor fibroids.

- HRNet relies on a parallel structure enabling the model to connect multi-resolution subnetworks in a novel and effective way. It starts from a high-resolution subnetwork as the first stage and gradually adds high-to-low resolution subnetworks one by one to form more stages, the multiresolution subnet- works being connected in parallel. The main difference is that HIFUNet and HRNet use different ways for computing high-resolution representation. Our HIFUNet employs the way of recovering highresolution representations from low-resolution representations outputted by a network (e.g. ResNet). While in HRNet, the authors propose another way that maintaining high-resolution representations through high-resolution convolutions and strengthening the representations with parallel low-resolution convolutions.

- U-Net uses a simple downsampling way to extract features while HIFUNet uses ResNet101 as the backbone to extract more features. We add large kernels in the skipconnections to increase the valid receptive field (VRF).

- CE-Net uses the Dense Atrous Convolution (DAC) module with multi-scale convolution and the Residual Multikernel Pooling (RMP) with multi-scale pooling at the bottom to extract and decode multi-scale features in parallel, as well directly integrate them. It ignores the global scene content at each level which further enhance the localization effect of the skip connection, as well as the progressivity and the correlativity among the multiscale structure.

Especially different from the CE-Net, the proposed HIFUNet adopts GCN in each skip connection between the encoder and the decoder. So that it is able to embed global scene information in the decoder, avoiding the global scene information loss in the dimension reduction during encoding. Besides, the HIFUNet also employs DMAC with the series structure and hierarchical fusion at the bottom of the encoder to progressively and correlatively extract multi-scale structure for the semantic objects.

- AttentionUNet proposes a novel Attention Gate (AG) model for medical imaging that automatically learns to focus on target structures of varying shapes and sizes, which brings a risk of transmitting multiplicative error along with the network.

CE-Net and AttentionUNet are both based on the U-Net and keep the way of extracting features in the encoder of U-Net. Differently, we choose to use a ResNet-101 pretrained on ImageNet as our backbone, which can be easier to train ResNet than training simple deep convolutional neural networks and resolve the problem of accuracy degradation.

- LEDNet aims at real-time semantic image segmentation. It employs an asymmetric encoder-decoder architecture. The encoder adopts a ResNet as the backbone network, where two new operations, channel split and shuffle, are utilized in each residual block to greatly reduce the computational cost while maintaining a higher segmentation accuracy. On the other hand, an Attention Pyramid
TABLE I

THE SCAN PARAMETERS AND CHARACTERISTICS OF MR DATASETS

\begin{tabular}{c|c}
\hline Variable & Value \\
\hline Repetition time (TR) & $3040 \mathrm{~ms}$ \\
echo time (TE) & $107.5 \mathrm{~ms}$ \\
field of view (FOV) & $28 \times 22.4 \mathrm{~cm}$ \\
slice thickness & $6 \mathrm{~mm}$ \\
slice gap & $1 \mathrm{~mm}$ \\
matrix & $304 \times 304$ \\
age (years) & $40.8 \pm 6.6^{*}$ \\
\hline *Age is Meanvalue \pm S.D
\end{tabular}

Network (APN) is employed in the decoder to further decrease the entire network complexity.

In our task, we pay more attention to the segmentation accuracy than to the efficiency of training. In the decoder part, LEDNet focuses on the last feature map from the encoder network, while some low-level features can be let out, which is not conducive to recovering detailed information. Therefore, we choose to recover the highresolution information by concatenating low- and highlevel features, which can help to identify the objects of all sizes and the details in complex medical images.

\section{EXPERIMENT AND DISCUSSIONS}

\section{A. Datasets}

The preoperative fat-suppressed T2-weighted MR images in the sagittal direction from 297 patients were used in this work. These images were collected from the First Affiliated Hospital of Chongqing Medical University. Sagittal T2-weighted fast spin-echo images were performed using a 3.0T MR unit (Signa HD Excite, GE Healthcare, Marlborough, MA) with an eight-channel phased-array coil. The scan parameters and characteristics of MR images are shown in Table I

Each MR volume consists of 25 slices of $304 \times 304$ pixels. The ground truth has been generated through a proper annotation process. To ensure an objective and consistent clinical reference, two radiologists were solicited for consensus agreement. This procedure included three steps:

1) Annotations through discussions: The discussion between two radiologists A (7-year experience) and B (15-year experience) was held in a face-to-face mode to set the annotation rules and identify special and complicated cases. It appeared, in this application, that the variability of the annotations mainly exists on the contour of the cervix and some minor fibroids.

2) The radiologist A took 2 months in annotating (no more than 5 volumes per day). After annotating 10 volumes, a second face-to-face discussion was held to analyze the firstround annotation, and improve the annotation rule further.

3) Then the radiologist A processed all cases (297 patients). Radiologist B checked all results and marked the cases which have some divergent views. Then, they held a face-to-face discussion and solved these situations.

After the above three steps, a full agreement between the two radiologists was obtained.

The research associated with the treatment of uterine fibroids was approved by the ethics committee and has no implication on patient treatment. 


\section{B. Experimental Setup}

1) Training and testing phase: We used for training and testing MR images from 260 and 37 patients, respectively. The number of images in the testing set is 925 . The use of a small amount of training data can result in overfitting. To prevent overfitting due to the limited number of images, the training data were augmented by image manipulation [57]. We applied the random shifting and scaling strategies (zoom range of 0.1 , the shift of $0.5 \mathrm{~mm}$ ).

2) Parameter settings and platform: For the optimization of our network, we use the Adam optimizer and set the initial learning rate to 2e-4. After each epoch, if we observe that the validation loss does not decrease for three consecutive times, the learning rate is reduced to $1 / 5$ of its current value until it stops at 5e-7. Therefore, the number of training epochs is determined by the decreasing learning rate. The batch size is set to 8 . All the comparative experiments adopt the same strategy for updating the hyperparameters. Besides, in the ablation study, the hyperparameters are fixed when removing parts of the network.

Our proposed network is based on the pretrained ResNet101 model on ImageNet. Notice that we adapt the first convolution operation because, as mentioned in III-A, we have a single channel input image instead of RGB channels like in natural images. The implementation is carried out on the PyTorch platform. The training and testing bed are ubuntu 16.04 system with NVIDIA Titan XP GPU (12 GB memory) and CUDA 9.0.

\section{Evaluation Metrics}

Different quantitative measures are used to comprehensively evaluate and compare the segmentation performance with other methods. We use the area-based indexes to compare the predicted segmentation results with the ground-truth manually labeled by radiologists. These indexes include the Dice coefficient (DSC) [58], Precision [59], Sensitivity (SE) [60], Specificity (SP) [60], Jaccard index (JI) [61], False Positive Ratio (FPR), False Negative Ratio (FNR) and False Region Ratio (FRR) [40]. We also use the distance-based indexes to evaluate the segmentation in terms of the location and shape accuracy of the extracted region boundaries such as the Mean Absolute Distance (MAD [40], Maximum Distance (MAXD) [40] and Hausdorff Distance (HD) [62].

\section{Comparison with Conventional Methods and Discussion}

As mentioned in Section II-A, Rundo et al. [37] and Militello et al. [39] proposed to segment uterine fibroids after treatment and evaluated them in [40]. We compare their methods with our method on the same dataset (fat-suppressed T2-weighted MR images composed of 375 slices issued from 15 patients).

It can be noticed that the above two methods are based on the fact that ablated fibroids appear as homogeneous hypointense regions with respect to the rest of the uterus (after contrast medium injection). Before the treatment, all kinds of fibroids appear as different states, which makes the segmentation task harder. For all patients, area-based and distance-based indexes were computed based on a slice-by-slice comparison and were performed on each slice having a fibroid area. The results are displayed in Table II. They show the superiority of the proposed method over the other two approaches and demonstrate its ability for uterine fibroid segmentation.

Some visual results are depicted in Fig. 6. It can be seen that, in the Patient 4, the gray level values around the area outlined by the circles have little difference from adjacent tissue. While in the post-treatment MR images the ablated tissue does not absorb the contrast medium and is hypo-intense with respect to the uterus, the use of simple adaptive global thresholding and region growing methods remains possible. However, the quality of the MR images is affected by noise which may lead to gray values in the regions of uterine fibroids similar to those of the surrounding tissues. As it is shown in Patient 7, there are two fibroids that appear with different signal strengths because of the different moisture contents: one is dark and the other one is bright. Thus, it is difficult for IOTS to distinguish the two different grayscale distributions of fibroids. SM\&RG fails to identify the contour of fibroids and assimilates the uterus to fibroids. The segmentation provided by our DL method is close to the ground-truth segmented by the clinical experts.

Additional comments on the two methods used here for comparison deserve to be made. The uterus ROI segmentation is a preliminary step for a robust fibroid detection in [40]. This task can be accomplished manually by the user to remove parts outside the uterus which are present in sagittal sections [37] or can rely on the Fuzzy C-Means (FCM) [39], which is an automatic method but where the number of clusters is set according to a visual inspection (i.e. anatomical properties of the analyzed pelvic images by considering image features) and experimental evidence (by means of segmentation trials). It means that the intervention of the experts is indispensable and that a complex and time-consuming preprocessing is needed before applying the intensity-based clustering technique. In conclusion, although these conventional methods have some merits in terms of performance, they show some practical limits in the clinical setting.

\section{E. Comparison with Other Deep Learning Methods}

We compare our method with six state-of-the-art (SOTA) algorithms, including U-Net [6], Attention-Unet [20], GCN [21], CE-Net [52], HRNet [63], LEDNet [64]. Their original implementations were kept and the same experimental conditions were used.

We select four of these competitive methods (U-Net, GCN, HRNet and CE-Net) to visually compare our method in Fig. 7 where the segmentation results are overlaid on the raw images. Different colors denote different classes (red denotes the fibroids, blue the uterus and green the spine). The images show that our method provides more accurate results. The performance of the six selected methods is presented in Table III for quantitative comparison. Among them, HRNet is the best method for segmenting uterus and fibroids. Besides, for the spine which has a high contrast with adjacent tissues, the introduction of the attention mechanism (i.e. AttentionUNet) performs quite well. However, overall 
TABLE II

VALUES OF AREA-BASED AND DISTANCE-BASED FOR SEGMENTING UTERINE FIBROIDS USING DIFFERENT METHODS ON T2-WEIGHTED MR IMAGES

\begin{tabular}{|c|c|c|c|c|c|c|c|c|c|c|c|}
\hline \multirow{2}{*}{ Method } & \multicolumn{8}{|c|}{ area-based } & \multicolumn{3}{|c|}{ distance-based } \\
\hline & $\operatorname{DSC}(\%)$ & Precision(\%) & $\mathrm{SE}(\%)$ & $\mathrm{SP}(\%)$ & $\mathrm{JI}(\%)$ & FPR & FNR & FRR & MAD & MAXD & $\mathrm{HD}$ \\
\hline ITOS & 80.50 & 76.83 & 89.03 & 98.22 & 69.34 & 0.018 & 0.110 & 0.540 & 2.432 & 7.893 & 8.893 \\
\hline $\mathrm{SM} \& \mathrm{~F}$ & 1.15 & 77.74 & 89.47 & 98.33 & 72.13 & 0.017 & 0.105 & 0.42 & 3.422 & 11.536 & 12.935 \\
\hline Proposed & 86.58 & 88.17 & 88.45 & 99.53 & 78.45 & 0.005 & 0.116 & 0.709 & 2.955 & 9.365 & 16.372 \\
\hline
\end{tabular}

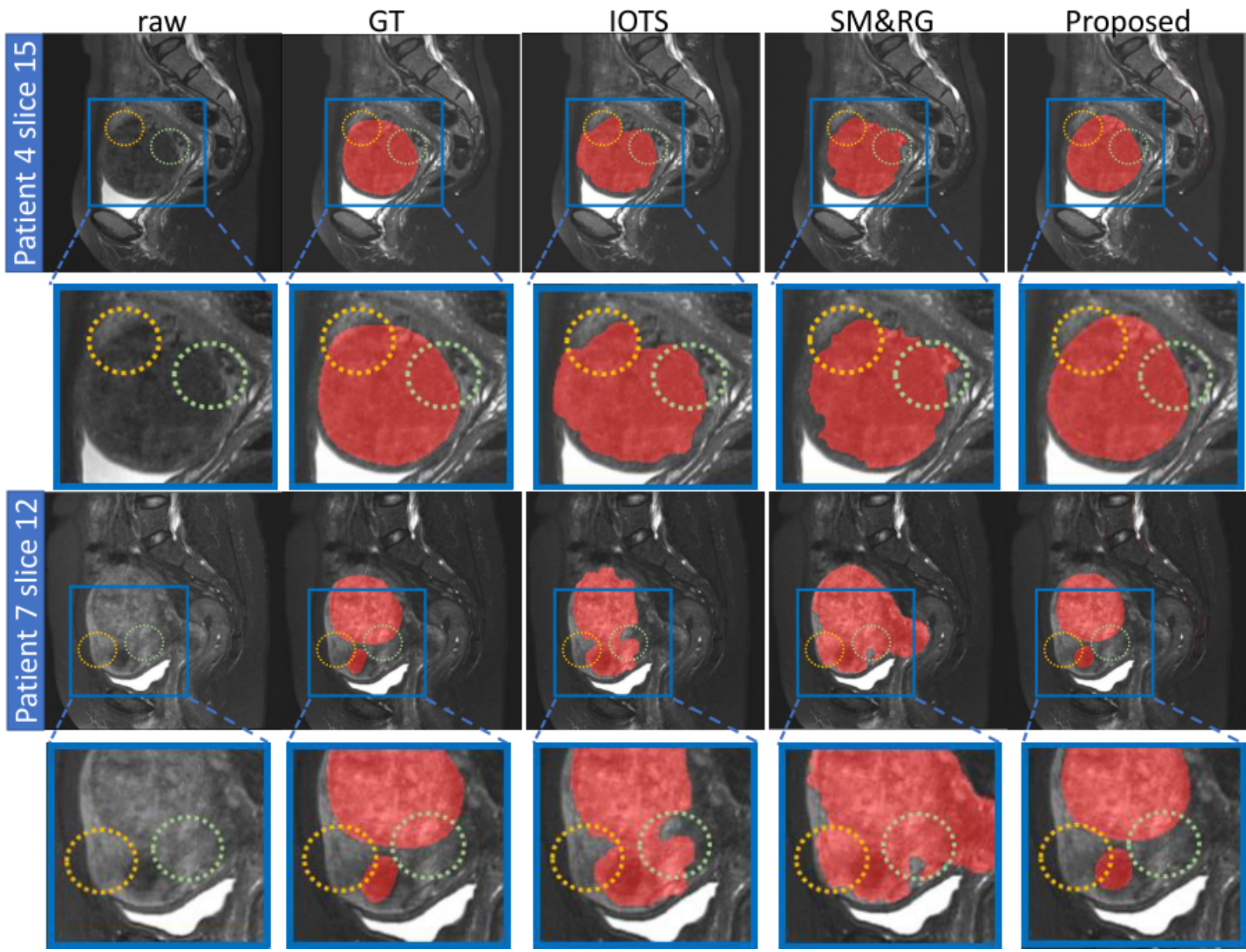

Fig. 6. Visualization of the segmentation results of uterine fibroids by using the proposed method and methods in [37], [39] for two patients. Red denotes the fibroids, and the yellow and green circles point out incorrect segmentation of uterine fibroids due to the little gray value difference with surrounding tissues

the best results are obtained by our method. Regarding the computation cost, we choose the GPU memory requirements and the test time for evaluating each slice to evaluate. Because of using ResNet as our backbone, our HIFUNet has a larger number of parameters. However, in clinical applications, the accuracy of the segmentation is much more important than the computation cost. From Table III, we can see that the performance of HIFUNet is significantly better in comparison to the other methods. We found it acceptable that the increases in computational costs are negligible for the improvement in accuracy. The computational cost of our method at test time can be borne by a standard GPU.

As can be seen from Fig. 7, the fibroids are more difficult to segment than the uterus, due to their unclear boundaries and undefined shapes. For patient 9, GCN and HRNet fail to segment the spine. For patient 8, U-Net, HRNet and CE-Net lead to incomplete segmentations. We can also observe the crucial role of the large receptive field used in our approach. Fig. 8 and Fig. 9 show the DSC of uterus and fibroid segmentation results in the form of box plots. Our method provides the best and steadiest performance in segmenting both uterus and fibroids while the performance of HRNet is slightly weaker.

\section{F. Ablation Study}

1) DMAC block: We first conducted ablation studies and validated the effectiveness of our DMAC block using the same training strategy and datasets. The original GCN (GCN-no DMAC [21]) was compared with the modified GCN (GCNDMAC) with a DMAC block added in the last layer. In the proposed HIFUNet (Proposed-DMAC), the DMAC block was put in the last layer and before the operation of global convolution. Comparisons were performed between the ProposedDMAC, removal of DMAC block (Proposed-no DMAC) and 


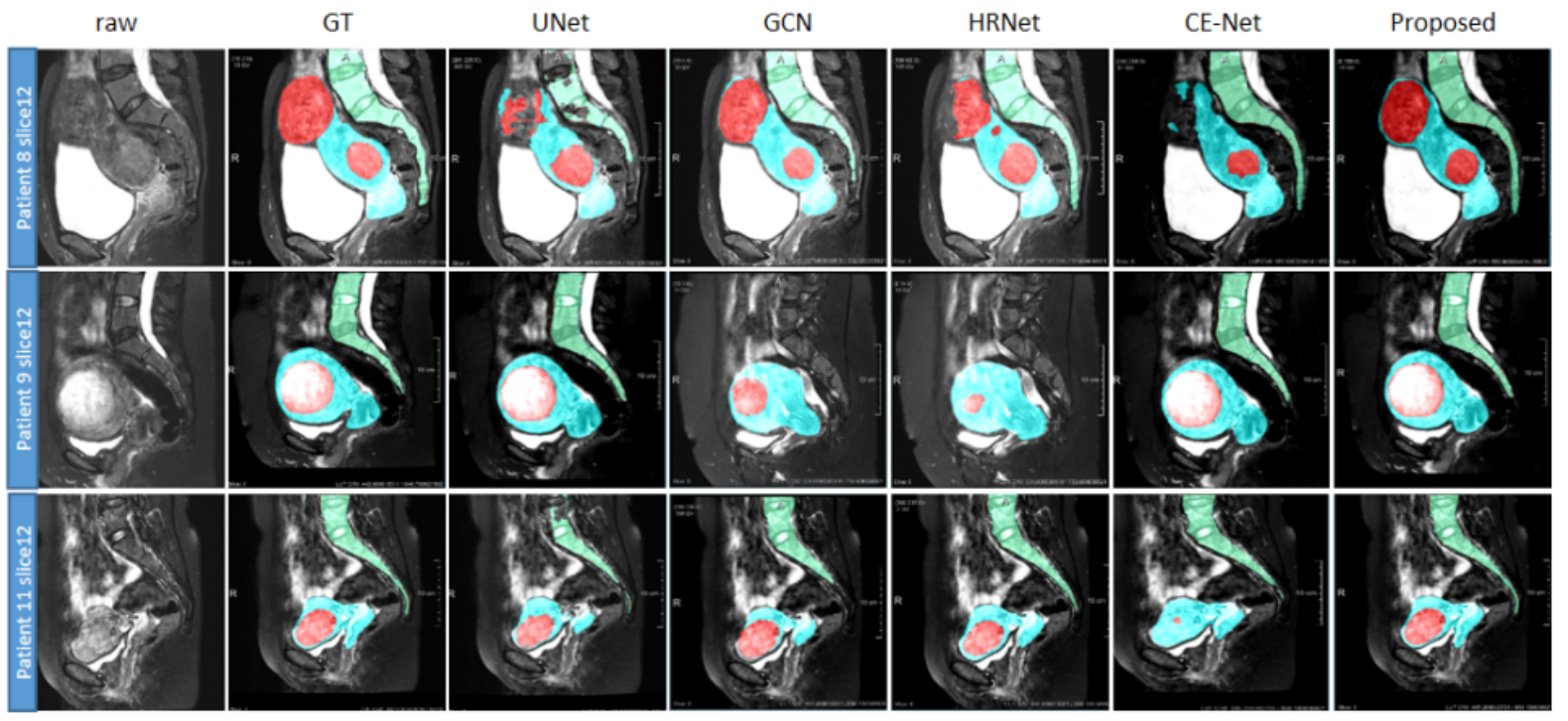

Fig. 7. Visualization of the segmentation results of uterus, fibroids and spine by using the proposed method and other four SOTA methods. From top to bottom are three different patients. Red denotes the fibroids, blue denotes uterus, and green denotes spine

TABLE III

QUANTITATIVE COMPARISON OF THREE EVALUATION INDEXES OF DIFFERENT SEGMENTATION METHODS ON TESTING DATASET (THE BEST RESULTS ARE INDICATED IN BOLD)

\begin{tabular}{|c|c|c|c|c|c|c|c|c|c|c|c|}
\hline \multirow{2}{*}{ Method } & \multicolumn{3}{|c|}{ Uterus } & \multicolumn{3}{|c|}{ Fibroid } & \multicolumn{3}{|c|}{ Spine } & \multirow{2}{*}{ Memory } & \multirow{2}{*}{ Test time } \\
\hline & DSC & Precision & Recall & DSC & Precision & Recall & DSC & Precision & Recall & & \\
\hline GCN [21] & $79.44 \%$ & $79.27 \%$ & $80.37 \%$ & $80.43 \%$ & $82.88 \%$ & $80.04 \%$ & $80.50 \%$ & $85.14 \%$ & $77.74 \%$ & $464.96 \mathrm{M}$ & $108.25 \mathrm{~ms}$ \\
\hline HRN & $80.43 \%$ & 78.2 & $83.45 \%$ & $80.88 \%$ & $85.39 \%$ & 80.7 & $85.45 \%$ & & $86.50 \%$ & & $55 \mathrm{~ms}$ \\
\hline & $34 \%$ & & 74.8 & 77.5 & $78.39 \%$ & 79.2 & $\%$ & 89. & & & Gms \\
\hline CE-N & $74.69 \%$ & $75.42 \%$ & $74.99 \%$ & $76.38 \%$ & $75.05 \%$ & $80.66 \%$ & $82.48 \%$ & $86.99 \%$ & $79.15 \%$ & $123.22 \mathrm{M}$ & $105.77 \mathrm{~ms}$ \\
\hline AttentionUNet [20] & $74.79 \%$ & $76.08 \%$ & $74.56 \%$ & $76.24 \%$ & $74.97 \%$ & $81.18 \%$ & $83.28 \%$ & $88.54 \%$ & $79.25 \%$ & $927.34 \mathrm{M}$ & $159.12 \mathrm{~ms}$ \\
\hline LEDNet [64] & $77.87 \%$ & $77.10 \%$ & $79.46 \%$ & $78.92 \%$ & $83.71 \%$ & $76.12 \%$ & $79.02 \%$ & $87.19 \%$ & $74.19 \%$ & 121.37M & $73.84 \mathrm{~ms}$ \\
\hline Proposed & $82.37 \%$ & $\mathbf{7 9 . 4 5 \%}$ & $86.00 \%$ & $83.51 \%$ & $84.48 \%$ & $83.70 \%$ & $85.01 \%$ & $82.51 \%$ & $88.69 \%$ & $503.71 \mathrm{M}$ & $109.83 \mathrm{~ms}$ \\
\hline
\end{tabular}

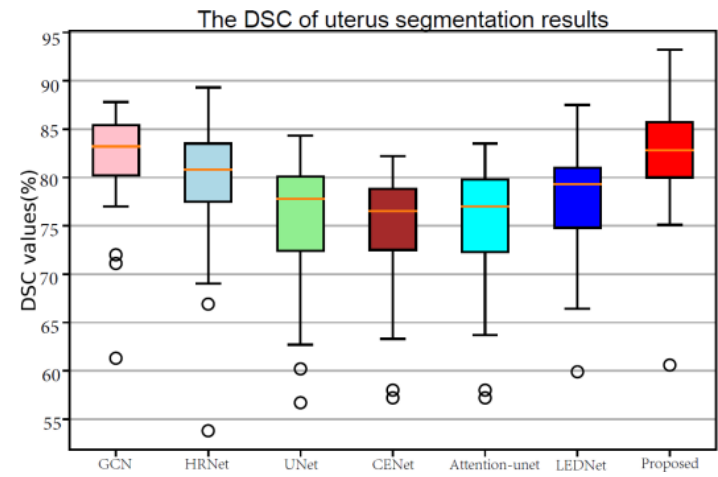

Fig. 8. The qualitative uterus segmentation performance is presented as boxplots. The $\mathrm{y}$ axis indicates the DSC values, while the $\mathrm{x}$ axis corresponds to the different methods (Unfilled circles denote the suspected outliers)

insertion of the DMAC after the global convolutional operation (Proposed-DMAC behind). Table IV shows the results of this study together with the time needed for each training epoch. They point out that the segmentation results are not significantly improved for GCN-DMAC. Concerning the DMAC position in our method, the computation time is strongly reduced when it is behind but the performance is worse than DMAC in-front (i.e. Proposed-DMAC).

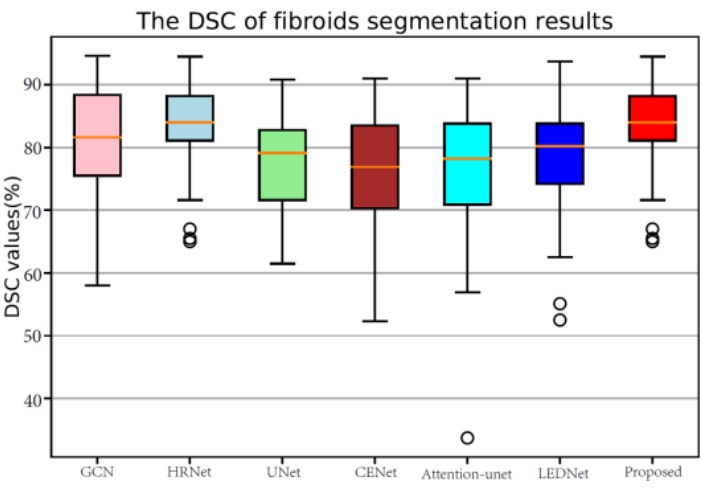

Fig. 9. The qualitative fibroid segmentation performance is presented as boxplots. The $\mathrm{y}$ axis indicates the DSC values, while the $\mathrm{x}$ axis specifies the different methods (Unfilled circles denote the suspected outliers)

Some images are shown in Fig. 10 for visual inspection. GCN leads to a relatively good segmentation of uterus and spine but the boundary of the fibroids is clearly inaccurate, and most parts of the fibroids fail to be labeled out. Adding the DMAC helps to refine the inaccurate boundary of the uterus and correct to some extent the wrong segmentation of fibroids. When replacing GCN by our proposed main structure, two fibroids are labeled out successfully with accurate boundaries 
TABLE IV

THE MEAN DSC AND COMPUTATION TIME OF DIFFERENT SEGMENTATION METHODS USING DMAC BLOCK (THE BEST RESULTS ARE INDICATED IN BOLD)

\begin{tabular}{ccccc}
\hline Method & Uterus & Fibroids & Spine & Time $(\mathrm{s})$ \\
\hline GCN-no DMAC & $79.44 \%$ & $80.43 \%$ & $80.50 \%$ & 164 \\
GCN-DMAC & $80.15 \%$ & $81.08 \%$ & $80.01 \%$ & $\mathbf{1 6 1}$ \\
Proposed-no DMAC & $76.87 \%$ & $78.84 \%$ & $84.28 \%$ & 479 \\
Proposed-DMAC behind & $77.72 \%$ & $77.47 \%$ & $80.89 \%$ & 441 \\
Proposed-DMAC & $\mathbf{8 2 . 3 7 \%}$ & $\mathbf{8 3 . 5 1 \%}$ & $\mathbf{8 5 . 0 1 \%}$ & 1094 \\
\hline
\end{tabular}
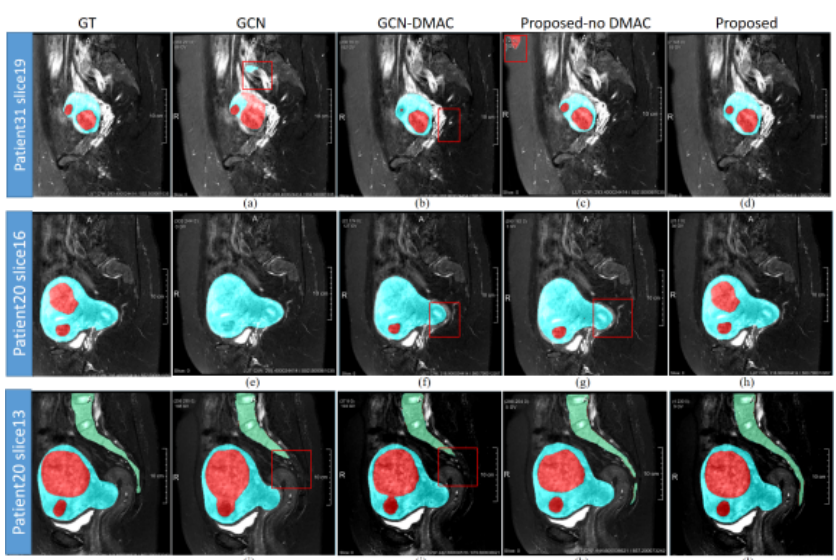

Fig. 10. Visualization of the segmentation results of uterus, fibroids and spine from two patients by using different methods which are mentioned in Table IV From left to right: ground-truth, GCN [21], GCN with DMAC, our proposed method without/with DMAC. Red denotes the fibroids, blue denotes uterus, and green denotes spine. Details are drawn by red box to see apparently

(see Patient 20 slice 13) which shows the advantage of our main structure. In the same slice, by comparing GCN and the Proposed-no DMAC, the boundary of the spine is corrected, which confirms the previous observation. A slightly better result can be achieved with DMAC. In all cases, our method labels both the uterus and the inside fibroids accurately which shows the effectiveness of the proposed DMAC. In particular, by comparing the last two columns, we can conclude that DMAC can extract the features of a large receptive field in a multi-scale context from multi-level feature maps.

2) Decoder method: In our approach, we replace the summation operation in GCN by a concatenation operation in UNet. Besides, in the procedure of upsampling, the deconvolution operation is employed to recover the original image size and to get the output mask. Recent contributions focus on the use of an upsampling module to upsample a low-resolution feature map given high-resolution feature maps as guidance. For instance, Joint Pyramid Upsampling (JPU) [65] aims at generating a high-resolution target image by transferring details and structures from the guidance image. Dupsampling (DUP) [66] was also proposed to replace the standard bilinear upsampling to recover the final pixel-wise prediction. The DUP takes advantage of the redundancy in the label space of semantic segmentation and is able to recover the pixel-wise prediction from low-resolution outputs of CNNs.

We report here the experiments made in order to compare different ways of decoding. Inspired by Octave Convolu- tion [67], in which Chen proposed to store and process lowfrequency and high-frequency characteristics respectively, we plan to deal with low and high channels separately. Also motivated by the Inception module [68] which employed a split-transform-merge strategy, we design a Channel-Split module that splits channels of each feature map after the GCN module into high and low channels and then we use concatenation and summation operations to integrate features of different layers in a continuous way. Different from Octave Convolution in [67] which is an operation as a direct replacement of vanilla convolutions, Channel-Split (CS) is a decoder strategy to change the way of merging different channels from different layers. Another decoding method is shown in Fig. 2. It removes the operations of summation in each layer and mainly uses deconvolution and concatenation. We name it Concatenation-Decoding (CD).

We train the three networks, with JPU or CS or CD as decoder respectively. The backbone here is the encoder of ResNet101 with GCN block and DMAC block. DUP is not trained because there is no formal code implementation of it. The comparison experiments are based on the same training parameter settings over the same training and validation dataset. The quantitative assessment is performed on the same testing dataset. The implementation of the JPU refers to the official PyTorch version on https://github.com/wuhuikai/FastFCN.

As it is shown in Table V, CD is much better than JPU and CS methods, with a benefit in DSC varying between $6 \%$ and $16 \%$ for uterus. It can be concluded that concatenation helps to recover the features especially in complex contexts and multiple targets. The summation is applied in the shortcuts (skip connections) in ResNet. It can help the network to speed up the training process and improve the gradient flow since the shortcuts are taken from previous convolution operations. Therefore, it is effective for the backpropagation to transfer error corrections to earlier layers, which can address the problem of vanishing gradient. However, due to the summation of the different channels or feature maps in CS, it may be difficult for the networks to distinguish different targets or recover the object details in the decoder. In contrast, the concatenation in $\mathrm{CD}$ operates on the feature maps generated by different filter sizes and keeps the information of different resolution feature maps since the information of features is not lost by summing up. JPU mainly uses the last three layers in the encoder. Therefore, the features of multiple objects in our complicated context may not be fully exploited by employing JPU.

\section{CONCLUSION}

In this paper, we have proposed a global convolutional network with deep multiple atrous convolutions to segment uterus, uterine fibroids and spine automatically. The employment of the DMAC block allows capturing effectively more low and high-level features.

Experimental results on the same datasets and platform demonstrated (i) the accuracy and robustness of the proposed method, (ii) a significant improvement when compared to 
TABLE V

THE PERFORMANCE ON TESTING DATASET BY USING DIFFERENT DECODER METHODS (THE BEST RESULTS ARE INDICATED IN BOLD)

\begin{tabular}{|c|c|c|c|c|c|c|c|c|c|}
\hline \multirow{2}{*}{ Method } & \multicolumn{3}{|c|}{ Uterus } & \multicolumn{3}{|c|}{ Fibroid } & \multicolumn{3}{|c|}{ Spine } \\
\hline & DSC & Precision & Recall & DSC & Precision & Recall & DSC & Precision & Recall \\
\hline Backbone+JPU & $66.26 \%$ & $70.44 \%$ & $63.37 \%$ & $67.36 \%$ & $70.20 \%$ & $66.74 \%$ & $66.07 \%$ & $76.90 \%$ & $58.79 \%$ \\
\hline Backbone+CS & $76.37 \%$ & $80.77 \%$ & $73.27 \%$ & $80.06 \%$ & $79.55 \%$ & $83.31 \%$ & $83.88 \%$ & $87.45 \%$ & $81.37 \%$ \\
\hline Backbone+CD (Proposed) & $82.37 \%$ & $79.45 \%$ & $86.00 \%$ & $83.51 \%$ & $84.48 \%$ & $83.70 \%$ & $85.01 \%$ & $82.51 \%$ & $88.69 \%$ \\
\hline
\end{tabular}

state-of-the-art segmentation methods and (iii) the performance could be close to radiologist level.

Although the proposed method shows promising results, some boundary inaccuracies may still be present in patients depicting multiple fibroids (see the left fibroid in the first row of Fig. 10). We plan to improve our approach by working directly in 3D (i.e. 3D convolutional filters) instead of dealing with 2D slices. This will make the training issues (improving efficiency and reducing training time) more critical. Other ideas should also be explored such as the use of prior anatomical and pathological knowledge on the uterus and spine. Coupling our approach with other techniques (active contour models, for instance) to refine the boundaries of the uterus and spine may also offer a sound way to correct the remaining errors mentioned above.

\section{ACKNOWLEDGMENT}

This research was supported by National Natural Science Foundation under grants (31571001, 61828101, 61876037), the National Key Research and Development Program of China (2017YFC0107903), the Excellence Project Funds of Southeast University and the Science Foundation for The Excellent Youth Scholars of Southeast University. .

\section{REFERENCES}

[1] E. A. Stewart, "Uterine fibroids," The Lancet, vol. 357, no. 9252, pp. 293-298, jan 2001.

[2] D. Shen, G. Wu, and H.-I. Suk, "Deep learning in medical image analysis," Апnи Rev Biomed Eng, vol. 19, no. 1, pp. 221-248, jun 2017.

[3] G. Litjens, T. Kooi, B. E. Bejnordi, A. A. A. Setio, F. Ciompi, M. Ghafoorian, J. A. Van Der Laak, B. Van Ginneken, and C. I. Sánchez, "A survey on deep learning in medical image analysis," Med Image Anal, vol. 42, pp. 60-88, dec 2017.

[4] R. Ge, G. Yang, Y. Chen, L. Luo, C. Feng, H. Ma, J. Ren, and S. Li, "K-Net: integrate left ventricle segmentation and direct quantification of paired echo sequence," IEEE Trans Med Imag, vol. 39, no. 5, pp. 1690-1702, may 2020.

[5] J. Long, E. Shelhamer, and T. Darrell, "Fully convolutional networks for semantic segmentation," in 2015 IEEE Conference on Computer Vision and Pattern Recognition (CVPR), Jun. 2015, pp. 3431-3440.

[6] O. Ronneberger, P. Fischer, and T. Brox, "U-Net: convolutional networks for biomedical image segmentation," in Medical Image Computing and Computer-Assisted Intervention - MICCAI 2015, ser. Lecture Notes in Computer Science, vol. 9351, 2015, pp. 234-241.

[7] Ö. Çiçek, A. Abdulkadir, S. S. Lienkamp, T. Brox, and O. Ronneberger, "3D U-Net: learning dense volumetric segmentation from sparse annotation," in Medical Image Computing and Computer-Assisted Intervention - MICCAI 2016, ser. Lecture Notes in Computer Science, vol. 9901, 2016, pp. 424-432.

[8] F. Milletari, N. Navab, and S.-A. Ahmadi, "V-Net: fully convolutional neural networks for volumetric medical image segmentation," in 2016 Fourth International Conference on 3D Vision (3DV), oct 2016, pp. 565571.

[9] X. Li, H. Chen, X. Qi, Q. Dou, C.-W. Fu, and P.-A. Heng, "HDenseUNet: hybrid densely connected UNet for liver and tumor segmentation from CT volumes," IEEE Trans Med Imag, vol. 37, no. 12, pp. 2663-2674, dec 2018.
[10] Z. Zhou, M. M. Rahman Siddiquee, N. Tajbakhsh, and J. Liang, "UNet++: a nested U-Net architecture for medical image segmentation," in Deep Learning in Medical Image Analysis and Multimodal Learning for Clinical Decision Support. DLMIA 2018, ser. Lecture Notes in Computer Science, vol. 11045, 2018, pp. 3-11.

[11] J. Zhang, Y. Jin, J. Xu, X. Xu, and Y. Zhang, "MDU-Net: multi-scale densely connected U-Net for biomedical image segmentation," in $\mathrm{ArXiV}$, no. 1812.00352, 2018.

[12] Q. Jin, Z. Meng, T. D. Pham, Q. Chen, L. Wei, and R. Su, "DUNet: a deformable network for retinal vessel segmentation," Knowledge-Based Systems, vol. 178, pp. 149-162, aug 2019.

[13] Q. Jin, Z. Meng, C. Sun, L. Wei, and R. Su, "RA-UNet: A hybrid deep attention-aware network to extract liver and tumor in CT scans," in $\mathrm{ArXiV}$, no. 1811.01328, 2018

[14] J. Dolz, I. B. Ayed, and C. Desrosiers, "Dense multi-path U-Net for ischemic stroke lesion segmentation in multiple image modalities," in Brainlesion: Glioma, Multiple Sclerosis, Stroke and Traumatic Brain Injuries. BrainLes 2018, ser. Lecture Notes in Computer Science, vol. 11383, 2019, pp. 271-282.

[15] J. Guo, J. Deng, N. Xue, and S. Zafeiriou, "Stacked dense U-Nets with dual transformers for robust face alignment," in ArXiV, no. 1812.01936, 2018.

[16] F. Isensee, J. Petersen, A. Klein, D. Zimmerer, P. F. Jaeger, S. Kohl, J. Wasserthal, G. Koehler, T. Norajitra, S. Wirkert, and K. H. Maier-Hein, "nnUu-net: self-adapting framework for U-Net-based medical image segmentation," in ArXiV, no. 1809.10486, 2018.

[17] A. Clèrigues, S. Valverde, J. Bernal, J. Freixenet, A. Oliver, and $\mathrm{X}$. Lladó, "Acute and sub-acute stroke lesion segmentation from multimodal MRI," in $\mathrm{ArXiV}$, no. 1810.13304, 2018.

[18] J. Dolz, C. Desrosiers, and I. B. Ayed, "IVD-net: intervertebral disc localization and segmentation in MRI with a multi-modal UNet," in Computational Methods and Clinical Applications for Spine Imaging. CSI 2018., ser. Lecture Notes in Computer Science, vol. 11397, 2019 pp. 130-143.

[19] J. Zhuang, "LadderNet: multi-path networks based on U-Net for medical image segmentation," in $\operatorname{ArXiV}$, no. 1810.07810, 2018.

[20] O. Oktay, J. Schlemper, L. L. Folgoc, M. Lee, M. Heinrich, K. Misawa, K. Mori, S. McDonagh, N. Y. Hammerla, B. Kainz, B. Glocker, and D. Rueckert, "Attention U-Net: learning where to look for the pancreas," in $\mathrm{ArXiV}$, no. 1804.03999, 2018.

[21] C. Peng, X. Zhang, G. Yu, G. Luo, and J. Sun, "Large kernel matters - improve semantic segmentation by global convolutional network," in IEEE Conference on Computer Vision and Pattern Recognition (CVPR), jul 2017, pp. 1743-1751.

[22] L.-C. Chen, G. Papandreou, I. Kokkinos, K. Murphy, and A. L. Yuille, "Semantic image segmentation with deep convolutional nets and fully connected CRFs," in arXiv, no. 1412.7062v4, 2014.

[23] — "DeepLab: semantic image segmentation with deep convolutional nets, atrous convolution, and fully connected CRFs," IEEE Trans Pattern Anal Mach Intell, vol. 40, no. 4, pp. 834-848, apr 2018.

[24] L.-C. Chen, Y. Zhu, G. Papandreou, F. Schroff, and H. Adam, "Encoderdecoder with atrous separable convolution for semantic image segmentation," in Computer Vision - ECCV 2018, ser. Lecture Notes in Computer Science, vol. 11211, 2018, pp. 833-851.

[25] F. Chollet, "Xception: deep learning with depthwise separable convolutions," in 2017 IEEE Conference on Computer Vision and Pattern Recognition (CVPR), jul 2017, pp. 1800-1807.

[26] L.-C. Chen, G. Papandreou, F. Schroff, and H. Adam, "Rethinking atrous convolution for semantic image segmentation," in arXiv, no. $1706.05587 \mathrm{v} 3,2017$.

[27] K. He, X. Zhang, S. Ren, and J. Sun, "Deep residual learning for image recognition," in 2016 IEEE Conference on Computer Vision and Pattern Recognition (CVPR), jun 2016, pp. 770-778.

[28] N. Ben-Zadok, T. Riklin-Raviv, and N. Kiryati, "Interactive level set segmentation for image-guided therapy," in 2009 IEEE International 
Symposium on Biomedical Imaging: From Nano to Macro, jun 2009, pp. 1079-1082.

[29] H. Khotanlou, A. Fallahi, M. A. Oghabian, and M. Pooyan, "Uterine fibroid segmentation on MRI based on Chan-Vese level set method and shape prior model," Biomedical Engineering: Applications, Basis and Communications, vol. 26, no. 02, p. 1450030, mar 2014.

[30] T. F. Chan and L. A. Vese, "Active contours without edges," IEEE Trans Image Process, vol. 10, no. 2, pp. 266-277, 2001.

[31] X. Bresson, P. Vandergheynst, and J.-P. Thiran, "A variational model for object segmentation using boundary information and shape prior driven by the Mumford-Shah functional," Int J Comput Vision, vol. 68, no. 2, pp. 145-162, mar 2006

[32] J. Yao, D. Chen, W. Lu, and A. Premkumar, "Uterine fibroid segmentation and volume measurement on MRI," in Medical Imaging 2006: Physiology, Function, and Structure from Medical Images, ser. Proc. SPIE, vol. 6143. SPIE, 2006, p. 614322.

[33] A. Fallahi, M. Pooyan, H. Ghanaati, M. A. Oghabian, H. Khotanlou, M. Shakiba, A. H. Jalali, and K. Firouznia, "Uterine segmentation and volume measurement in uterine fibroid patients' MRI using fuzzy C-Mean algorithm and morphological operations," Iranian Journal of Radiology, vol. 08, no. 03, pp. 150-156, dec 2011.

[34] A. Fallahi, M. Pooyan, H. Khotanlou, H. Hashemi, K. Firouznia, and M. A. Oghabian, "Uterine fibroid segmentation on multiplan MRI using FCM, MPFCM and morphological operations," in 2010 2nd International Conference on Computer Engineering and Technology, vol. 7, 2010, pp. V7-1-V7-5.

[35] L. Ma and R. C. Staunton, "A modified fuzzy C-means image segmentation algorithm for use with uneven illumination patterns," Pattern Recognition, vol. 40, no. 11, pp. 3005-3011, nov 2007.

[36] C. Militello, S. Vitabile, G. Russo, G. Candiano, C. Gagliardo, M. Midiri, and M. C. Gilardi, "A semi-automatic multi-seed regiongrowing approach for uterine fibroids segmentation in MRgFUS treatment," in 2013 Seventh International Conference on Complex, Intelligent, and Software Intensive Systems, jul 2013, pp. 176-182.

[37] L. Rundo, C. Militello, S. Vitabile, C. Casarino, G. Russo, M. Midiri, and M. C. Gilardi, "Combining split-and-merge and multi-seed region growing algorithms for uterine fibroid segmentation in MRgFUS treatments," Med Biol Eng Comput, vol. 54, no. 7, pp. 1071-1084, nov 2015.

[38] K. Antila, H. J. Nieminen, R. B. Sequeiros, and G. Ehnholm, "Automatic segmentation for detecting uterine fibroid regions treated with MRguided high intensity focused ultrasound (MR-HIFU)," Med Phys, vol. 41, no. 7, p. 073502, jun 2014.

[39] C. Militello, S. Vitabile, L. Rundo, G. Russo, M. Midiri, and M. C. Gilardi, "A fully automatic 2D segmentation method for uterine fibroid in MRgFUS treatment evaluation," Comput Biol Med, vol. 62, pp. 277292, jul 2015.

[40] L. Rundo, C. Militello, A. Tangherloni, G. Russo, R. Lagalla, G. Mauri, M. C. Gilardi, and S. Vitabile, "Computer-assisted approaches for uterine fibroid segmentation in MRgFUS treatments: quantitative evaluation and clinical feasibility analysis," in Quantifying and Processing Biomedical and Behavioral Signals, aug 2018, pp. 229-241.

[41] Y. Kurata, M. Nishio, K. Fujimoto, M. Yakami, A. Kido, H. Isoda, and K. Togashi, "Automatic segmentation of uterus with malignant tumor on MRI using U-net," in Computed Assisted Radiology and Surgery (CARS), 2017

[42] Y. Kurata, M. Nishio, A. Kido, K. Fujimoto, M. Yakami, H. Isoda, and K. Togashi, "Automatic segmentation of the uterus on MRI using a convolutional neural network," Comput Biol Med, vol. 114, p. 103438, nov 2019.

[43] J. Liu, Y. Pan, M. Li, Z. Chen, L. Tang, C. Lu, and J. Wang, "Applications of deep learning to MRI images: A survey," Big Datc Mining and Analytics, vol. 1, no. 1, pp. 1-18, mar 2018.

[44] S. M. Anwar, M. Majid, A. Qayyum, M. Awais, M. Alnowami, and M. K. Khan, "Medical image analysis using convolutional neural networks: a review," J Med Syst, vol. 42, no. 11, oct 2018.

[45] F. Milletari, S.-A. Ahmadi, C. Kroll, A. Plate, V. Rozanski, J. Maiostre, J. Levin, O. Dietrich, B. Ertl-Wagner, K. Bötzel, and N. Navab, "HoughCNN: Deep learning for segmentation of deep brain regions in MRI and ultrasound," Comput Vision Image Understanding, vol. 164, pp. 92-102, nov 2017.

[46] S. Valverde, M. Cabezas, E. Roura, S. González-Villà, D. Pareto, J. C. Vilanova, L. Ramió-Torrentà, À. Rovira, A. Oliver, and X. Lladó, "Improving automated multiple sclerosis lesion segmentation with a cascaded 3D convolutional neural network approach," Neuroimage, vol. 155 , pp. 159-168, jul 2017.
[47] C. Wachinger, M. Reuter, and T. Klein, "DeepNAT: Deep convolutional neural network for segmenting neuroanatomy," NeuroImage, vol. 170, pp. 434-445, apr 2018.

[48] S. Trebeschi, J. J. M. van Griethuysen, D. M. J. Lambregts, M. J. Lahaye, C. Parmar, F. C. H. Bakers, N. H. G. M. Peters, R. G. H. Beets-Tan, and H. J. W. L. Aerts, "Deep learning for fully-automated localization and segmentation of rectal cancer on multiparametric MR," Sci Rep, vol. 7, no. 1 , jul 2017

[49] W. Zhang, R. Li, H. Deng, L. Wang, W. Lin, S. Ji, and D. Shen, "Deep convolutional neural networks for multi-modality isointense infant brain image segmentation," NeuroImage, vol. 108, pp. 214-224, mar 2015.

[50] J. Bernal, K. Kushibar, D. S. Asfaw, S. Valverde, A. Oliver, R. Martí, and $\mathrm{X}$. Lladó, "Deep convolutional neural networks for brain image analysis on magnetic resonance imaging: a review," Artif Intell Med, vol. 95, pp. 64-81, apr 2019.

[51] M. Avendi, A. Kheradvar, and H. Jafarkhani, "A combined deep-learning and deformable-model approach to fully automatic segmentation of the left ventricle in cardiac MRI," Med Image Anal, vol. 30, pp. 108-119, may 2016.

[52] Z. Gu, J. Cheng, H. Fu, K. Zhou, H. Hao, Y. Zhao, T. Zhang, S. Gao, and J. Liu, "CE-net: context encoder network for 2D medical image segmentation," IEEE Trans Med Imag, vol. 38, no. 10, pp. 2281-2292, oct 2019.

[53] C. Szegedy, S. Ioffe, V. Vanhoucke, and A. A. Alemi, "Inception-v4, Inception-ResNet and the impact of residual connections on learning," in Proc. of the Thirty-First AAAI Conference on Artificial Intelligence, 2017, pp. 4278-4284.

[54] M. Holschneider, R. Kronland-Martinet, J. Morlet, and P. Tchamitchian, "A real-time algorithm for signal analysis with the help of the wavelet transform," in Wavelets. Inverse problems and theoretical imaging, 1990, pp. 286-297.

[55] J.-M. Combes, A. Grossmann, and P. Tchamitchian, Eds., Wavelets Time-Frequency Methods and Phase Space Proceedings of the International Conference, ser. Inverse problems and theoretical imaging. Springer, 1990.

[56] P. Wang, P. Chen, Y. Yuan, D. Liu, Z. Huang, X. Hou, and G. Cottrell, "Understanding convolution for semantic segmentation," in 2018 IEEE Winter Conference on Applications of Computer Vision (WACV), mar 2018.

[57] Z. Hussain, F. Gimenez, D. Yi, and D. Rubin, "Differential data augmentation techniques for medical imaging classification tasks." in AMIA ... Annual Symposium proceedings, vol. 2017, 2017, pp. 979-984.

[58] L. R. Dice, "Measures of the amount of ecologic association between species," Ecology, vol. 26, no. 3, pp. 297-302, 1945.

[59] F. C. Monteiro and A. C. Campilho, "Performance evaluation of image segmentation," in Image Analysis and Recognition. ICIAR 2006, ser. Lecture Notes in Computer Science, vol. 4141, 2006, pp. 248-259.

[60] R. Trevethan, "Sensitivity, specificity, and predictive values: foundations, pliabilities, and pitfalls in research and practice," Front Public Health, vol. 5, nov 2017.

[61] P. Jaccard, "The distribution of the flora in the alpine zone," New Phytol, vol. 11 , no. 2, pp. 37-50, feb 1912.

[62] J. Henrikson, "Completeness and total boundedness of the Hausdorff metric," MIT Undergraduate Journal of Mathematics, 1999.

[63] K. Sun, Y. Zhao, B. Jiang, T. Cheng, B. Xiao, D. Liu, Y. Mu, X. Wang, W. Liu, and J. Wang, "High-resolution representations for labeling pixels and regions," in ArXiV, no. 1904.04514v1, 2019.

[64] Y. Wang, Q. Zhou, J. Liu, J. Xiong, G. Gao, X. Wu, and L. J. Latecki, "LEDNet: a lightweight encoder-decoder network for real-time semantic segmentation," in $\mathrm{ArXiV}$, no. 1905.02423v3, 2019.

[65] H. Wu, J. Zhang, K. Huang, K. Liang, and Y. Yu, "FastFCN: rethinking dilated convolution in the backbone for semantic segmentation," in ArXiV, no. 1903.11816v1, 2019.

[66] Z. Tian, T. He, C. Shen, and Y. Yan, "Decoders matter for semantic segmentation: data-dependent decoding enables flexible feature aggregation," in 2019 IEEE/CVF Conference on Computer Vision and Pattern Recognition (CVPR), jun 2019, pp. 3121-3130.

[67] Y. Chen, H. Fan, B. Xu, Z. Yan, Y. Kalantidis, M. Rohrbach, S. Yan, and J. Feng, "Drop an Octave: Reducing spatial redundancy in convolutional neural networks with Octave convolution," in arXiv, no. 1904.05049v3, 2019.

[68] C. Szegedy, V. Vanhoucke, S. Ioffe, J. Shlens, and Z. Wojna, "Rethinking the Inception architecture for computer vision," in 2016 IEEE Conference on Computer Vision and Pattern Recognition (CVPR), jun 2016, pp. $2818-2826$ 